\title{
Splenic irradiation before allogeneic stem cell transplantation for myelofibrosis
}

\author{
Grzegorz Helbig $^{1}{ }^{10} \cdot$ Agata Wieczorkiewicz-Kabut $^{1} \cdot$ Mirosław Markiewicz ${ }^{1} \cdot$ Helena Krzemień $^{1}$. \\ Michał Wójciak $^{2} \cdot$ Krzysztof Białas $^{1} \cdot$ Małgorzata Kopera $^{1} \cdot$ Ewa Rzenno $^{1} \cdot \mathrm{Krzysztof} \mathrm{Woźniczka}^{1}$ Anna Kopińska ${ }^{1}$. \\ Iwona Grygoruk-Wiśniowska ${ }^{1} \cdot$ Anna Koclęga ${ }^{1}$
}

Received: 14 November 2018 / Accepted: 2 January 2019 / Published online: 8 January 2019

(c) The Author(s) 2019

\begin{abstract}
Splenectomy before allogeneic stem cell transplantation (ASCT) for patients with myelofibrosis (MF) remains a matter of debate, and conflicting results have been reported to date. The procedure seems to fasten post-transplant hematological recovery, but it does not have an impact on survival. The role of pre-transplant splenic irradiation (SI) is much more difficult to evaluate. Forty-four patients (25 males and 19 females) with MF at median age of 49 years at diagnosis (range 14-67) underwent ASCT. The post-transplant outcome was compared between irradiated and non-irradiated patients. Eleven patients received irradiation before transplantation. Median dose of radiation was $1000 \mathrm{cGy}$ (range 600-2400). There was no difference in median time to engraftment between patients with and without previous radiotherapy. Acute and chronic graft versus host disease (GVHD) occurred in $47 \%$ and $36 \%$ of patients, respectively. There was no difference in GVHD incidence between groups. Eight patients relapsed/progressed in irradiated group versus 17 in non-irradiated (70\% vs. 51\%; $p=0.3$ ). Transformation to acute myeloid leukemia was observed in 3 patients: 2 in irradiated and 1 in non-irradiated group. In total, 22 patients died with no statistical difference in death rate between irradiated and non-irradiated subjects. The probability of overall survival after transplant for the entire cohort at 2 years was $54 \%$ ( $72 \%$ for irradiated and $48 \%$ for non-irradiated patients; $p=0.25$ ). Splenic irradiation prior to ASCT for myelofibrosis has not beneficial effect on post-transplant outcome.
\end{abstract}

Keywords Myelofibrosis · Splenomegaly · Splenectomy · Splenic irradiation · Allogeneic stem cell transplantation · Outcome

\section{Introduction}

Myelofibrosis (MF) is a BCR-ABL-negative myeloproliferative neoplasm associated with bone marrow fibrosis and extramedullary hematopoiesis. Most patients carry JAK2, CALR or MPL mutation; however, none of these abnormalities are specific and essential for diagnosis of MF. Clinical manifestations of MF are variable; one-third of patents are asymptomatic at presentation, the remaining have symptoms

Grzegorz Helbig

ghelbig@o2.pl

1 Department of Hematology and Bone Marrow Transplantation, School of Medicine in Katowice, Medical University of Silesia, Dąbrowski Street 25, 40-032 Katowice, Poland

2 Students' Research Group, Medical University of Warsaw, Warsaw, Poland resulting mainly from anemia and splenomegaly (SM) [1]. The latter finding is a consequence of extramedullary hematopoiesis. SM is found in $90 \%$ of MF patients at initial presentation, and its size increases with disease duration producing pain in left upper quadrant and early satiety [2].

To date, only allogeneic stem cell transplantation (ASCT) remains the only curative option for patients with MF. However, due to high transplant-related mortality, ASCT is limited to small proportion of MF patients, usually for those at younger age at transplant, without significant co-morbidities and at higher prognostic categories [3, 4].

A pre-transplant splenectomy remains a matter of debate, and conflicting results have been reported to date. The procedure seems to fasten post-transplant hematological recovery, but its impact on relapse rate and survival is unclear. A trend for faster leukocyte engraftment but a significant higher incidence of relapse at 3 years was demonstrated for splenectomized MF patients in a large analysis of EBMT 
group. Of note is, that splenectomy had no impact on overall survival [3]. A French Study has confirmed the beneficial effect of splenectomy on post-transplant engraftment. Moreover, splenectomy in men had favorable impact on overall survival [5]. Pre-transplant splenectomy in MF patients was not associated with post-transplant relapse but with a prolonged overall and event-free survival in a recent published study [6].

The role of pre-transplant splenic irradiation (SI) in MF patients is much more difficult to evaluate. It was demonstrated in patients transplanted for chronic myeloid leukemia that SI before ASCT did not increase relapse incidence and transplant-related mortality [7]. There are only few small studies on SI prior to ASCT in MF patients [8,9].

In this paper, we report on the effects of pre-transplant SI on outcome of MF patients undergoing ASCT.

\section{Materials and methods}

The study patients were identified through the use of our institutional database of medical records. The diagnosis of MF was established according to WHO criteria [10]. Data on cytogenetic results were not reported as dry aspirates were present in a majority of patients at MF diagnosis, and thus, metaphases were not obtained. Risk stratification was performed according to the Dynamic International Prognostic Scoring System (DIPSS) [11]. Spleen size before and after ASCT was assessed using palpation and abdominal ultrasound. SI was indicated for patients with markedly enlarged splenomegaly at the discretion of treating physician. Doses and fractionation of splenic irradiation was individually based. All patients provided an informed consent in accordance with the Declaration of Helsinki.

Nonparametric comparisons of group means were performed by using the Mann-Whitney $U$ test. Proportions were compared by Fisher exact test. The distribution for overall survival (OS) was estimated using the method of Kaplan and Meier and compared using the log-rank test. A $p$ value less than 0.05 was considered significant. All computations were performed with StatSoft Poland analysis software (version 10.0).

\section{Results}

\section{Patient characteristics}

Forty-four patients (25 males and 19 females) with MF at median age of 49 years at diagnosis (range 14-67) underwent ASCT between 2004 and 2017. The diagnosis was as follows: primary myelofibrosis (PMF; $n=30$ ), postpolycythemia vera myelofibrosis (post-PV MF; $n=10$ ) and post-essential thrombocythemia myelofibrosis (post-ET MF; $n=4)$. According to the DIPSS, 3 patients were stratified to low risk group, 3 to intermediate-1, 8 to intermediate- 2 and 29 to high. The JAK2 V617F point mutation was detected in 29 patients (66\%), 12 patients were negative, and data were missing for one. $50 \%$ of studied patients were red blood cells (RBCs) transfusion-dependent at the time of diagnosis. Median number of treatment lines was 1 (range 0-5). Most frequent pre-transplant treatments included hydroxyurea $(n=23)$, corticosteroids $(n=15)$, thalidomide $(n=7)$, interferon $\alpha(n=5)$, androgens $(n=5)$. The other therapeutic options included anagrelide $(n=4)$, ruxolitinib $(n=2)$ and intensive chemotherapy $(n=2)$.

Eleven patients received splenic radiotherapy before transplant. Median time between SI and ASCT was 1.5 months (range 0.5-12). Median dose of radiation was $1000 \mathrm{cGy}$ (range 600-2400) administered in a median of 8 fractions (range 6-15). There was tendency to greater spleen size in irradiated patients $(p=0.06)$. Decrease in spleen size was demonstrated in 4 out of 11 (36\%) patients after SI. No severe complications of SI were observed. Patients' characteristics at diagnosis according to previous radiotherapy are shown in Table 1.

Median time from diagnosis to transplant was significantly shorter for patients without pre-transplant splenic irradiation when compared with those who underwent radiotherapy: 41 months (range 2-643) versus 122 months $(18-210) ; p=0.02$.

\section{Transplant data}

Median age at transplant was comparable between two studied groups ( 51 vs. 52 years; $p=0.7$ ). Blood parameters did not differ except hemoglobin level which was statistically higher for patients undergoing radiotherapy. Dynamic IPSS was intermediate- 2 or high in $84 \%$ of transplanted patients. Approximately $50 \%$ of patients remained RBCs and platelets transfusion-dependent at the time of transplantation. Constitutional symptoms were present in 20 out of 44 included subjects. Despite previous splenic radiotherapy, the pre-transplant spleen size measured on ultrasound was comparable between two groups of analyzed patients $(p=0.9)$. HLA identical sibling was available for 19 patients, the remaining received transplant from matched unrelated donors. Peripheral blood was a source of stem cells for all but one patient who received bone marrow. Reduced intensity conditioning consisted of busulphan and fludarabine was used in all but one patient who was treated with myeloablative regimen (busulphan and cyclophosphamide). Anti-thymocyte globulin (ATG) was administered in patients transplanted from unrelated donors. Graft versus host disease (GHVD) prophylaxis was uniform and included cyclosporine and methotrexate. 
Table 1 Comparison of patient characteristics at diagnosis according to previous radiotherapy

\begin{tabular}{|c|c|c|c|}
\hline Parameter & With radiotherapy & Without radiotherapy & $p$ value \\
\hline Number of patients & 11 & 33 & \\
\hline Gender (female/male) & $5 / 6$ & $14 / 19$ & 0.5 \\
\hline Age (median, range); years & $49(33-67)$ & $49(14-67)$ & 0.8 \\
\hline Primary disease $(n ; \%)$ & & & 0.39 \\
\hline Primary MF & $6(55)$ & $24(73)$ & \\
\hline Post-PV MF & $3(27)$ & $7(21)$ & \\
\hline Post-ET MF & $2(18)$ & $2(6)$ & \\
\hline $\operatorname{IPSS}(n ; \%)$ & & & 0.14 \\
\hline Low & 0 & $3(9)$ & \\
\hline Intermediate-1 & 0 & $3(9)$ & \\
\hline Intermediate- 2 & $2(18)$ & $6(18)$ & \\
\hline High & $9(82)$ & $20(60)$ & \\
\hline$J A K 2$ V617F mutation $(n ; \%)$ & & & 0.59 \\
\hline Positive & $9(82)$ & $22(67)$ & \\
\hline Negative & $2(18)$ & $10(30)$ & \\
\hline Missing & 0 & $1(3)$ & \\
\hline Spleen size below costal margin $(\mathrm{cm})$ & $10(5-16)$ & $9(0-20)$ & 0.38 \\
\hline RBCs dependence $(n ; \%)$ & & & 0.46 \\
\hline Yes & $5(45)$ & $17(52)$ & \\
\hline No & $6(55)$ & $15(45)$ & \\
\hline Missing & 0 & $1(3)$ & \\
\hline Number of treatment lines before transplant & $1(0-5)$ & $1(0-3)$ & 0.5 \\
\hline
\end{tabular}

One exception was a patient with mild renal failure at transplant who received cyclosporine with mycophenolate. Median number of transplanted CD34-positive cells were comparable between irradiated and non-irradiated subjects.

All patients but one with early post-transplant death, engrafted and there was no difference in median time to engraftment between patients with and without previous radiotherapy. Only one death within the first 30 days after transplant was noted and it occurred in non-irradiated patient. In total, eight patients did not achieve platelet count $>20 \times 10^{9} / \mathrm{L}$ at the day of discharge.

More than $50 \%$ of all patients demonstrated infectious complications after transplant; however, there was no difference between irradiated and non-irradiated subjects. The most frequent infectious manifestations included pneumonia $(n=9)$, urinary tract infection $(n=7)$, bacteremia $(n=5)$, pneumocystis pneumonia $(n=4)$, pharyngitis $(n=3)$, diarrhea (C. difficile; $n=2$ ). CMV reactivation was observed in three patients. Three patients developed septic shock successfully treated with antibiotics. Fever of unknown origin was seen in four patients.

Acute and chronic GVHD occurred in $47 \%$ and $36 \%$ of patients, respectively. Acute GVHD grade III/IV was present in 2 irradiated and 1 non-irradiated patients. There was no difference in GVHD occurrence between both groups. Twenty-five patients developed disease relapse or progression and three of them received second or subsequent transplant. Eight patients relapsed/progressed in irradiated group versus 17 in non-irradiated (70\% vs. 51\%; $p=0.3$ ).

Transformation to acute myeloid leukemia was observed in three patients: two in irradiated and one in non-irradiated group.

In total, twenty-two patients died (4 in irradiated and 18 in non-irradiated). There was no statistical difference in death rate between irradiated and non-irradiated subjects ( $63 \%$ vs. $45 \% ; p=0.48$ ). One patient died within the first 30 days after transplant due to cerebral hemorrhage, six patients expired between +30 and +100 days after transplant and the remaining died at later stage.

Among four irradiated patients who expired, two died of severe GVHD with subsequent infectious complications (bacterial and viral). Chemotherapy-resistant acute myeloid leukemia developed in the remaining two subjects. In nonirradiated subgroup, all but one patient died of bilateral pneumonia (bacterial or fungal) associated with immunosuppressant-resistant GVHD and CMV reactivation.

Twenty-two patients (50\%) are alive at the last contact. Twenty subjects were found to have full donor chimerism, and results were not available for additional two. Median time from diagnosis to last contact reached 4.5 years (range 0.6-22.3), and median time from transplant to last contact was 9.9 months (range 0.7-113). The results were 
Table 2 Transplant data in irradiated and non-irradiated patients with myelofibrosis

\begin{tabular}{|c|c|c|c|}
\hline Parameter & With radiotherapy & Without radiotherapy & $p$ value \\
\hline Age (median, range); years & $51(38-68)$ & $52(22-69)$ & 0.7 \\
\hline WBC count $\left(\times 10^{9} / \mathrm{L}\right)$; median, range & $9.63(1.28-198.9)$ & $7.1(0.8-222.5)$ & 0.47 \\
\hline Hemoglobin $(\mathrm{g} / \mathrm{dL})$; median, range & $10.4(8.3-15.6)$ & $8.9(5.9-16.5)$ & 0.05 \\
\hline Platelet count $\left(10^{9} / \mathrm{L}\right)$; median, range & $130(18-690)$ & $97(16-674)$ & 0.1 \\
\hline DIPSS $(n ; \%)$ & & & 0.18 \\
\hline Low & 0 & $3(9)$ & \\
\hline Intermediate-1 & 0 & $3(9)$ & \\
\hline Intermediate-2 & $1(9)$ & $5(15)$ & \\
\hline High & $10(91)$ & $21(67)$ & \\
\hline PLT transfusion-dependence $(n ; \%)$ & & & 0.38 \\
\hline Yes & $3(27)$ & $12(36)$ & \\
\hline No & $8(63)$ & $19(54)$ & \\
\hline RBCs transfusion-dependence $(n)$ & & & 0.36 \\
\hline Yes & $5(45)$ & $19(63)$ & \\
\hline No & $6(55)$ & $14(37)$ & \\
\hline Constitutional symptoms $(n ; \%)$ & & & 0.59 \\
\hline Yes & $5(45)$ & $15(45)$ & \\
\hline No & $6(55)$ & $18(55)$ & \\
\hline Spleen size below costal margin $(\mathrm{cm})$ before transplant & $16(12-24)$ & $8(0-18)$ & 0.06 \\
\hline Spleen size below costal margin $(\mathrm{cm})$ on day +30 after transplant & $5(0-14)$ & $4(0-20)$ & 0.7 \\
\hline Type of donor $(n ; \%)$ & & & 0.05 \\
\hline HLA identical sibling & $2(18)$ & $17(52)$ & \\
\hline HLA matched unrelated & $9(82)$ & $16(48)$ & \\
\hline Number of transplanted CD34-positive cells; median, range & $5.9(1.25-8.32)$ & $6.5(1.08-13.01)$ & 0.32 \\
\hline ANC $>0.5\left(\times 10^{9} / \mathrm{L}\right)$; median, range & $15(13-18)$ & $15(11-29)$ & 0.7 \\
\hline PLT $>20\left(\times 10^{9} / \mathrm{L}\right) ;$ median, range & $26(10-47)$ & $18(10-67)$ & 0.14 \\
\hline Acute GVHD $(n ; \%)$ & $5 / 11(45)$ & $16 / 33(48)$ & 0.5 \\
\hline Chronic GVHD $(n ; \%)$ & $3 / 8(37)$ & $5 / 9(45)$ & 0.64 \\
\hline Infectious complications $(n ; \%)$ & $4(36)$ & $21(63)$ & 0.1 \\
\hline Relapse/progression $(n ; \%)$ & $8(70)$ & $17(51)$ & 0.3 \\
\hline Alive $(n ; \%)$ & $7(63)$ & $15(45)$ & 0.48 \\
\hline Median time from transplant to last contact (months); median, range & $11.5(5.9-86.9)$ & $10(0.7-113)$ & 0.6 \\
\hline Median time from diagnosis to last contact (years); median, range & $5.4(0.9-12.5)$ & $3.9(0.6-22.3)$ & 0.42 \\
\hline
\end{tabular}

comparable between analyzed groups. Transplant data are summarized in Table 2.

The probability of overall survival after transplant for the entire cohort at 2 years was $54 \%$ (72\% for irradiated and $48 \%$ for non-irradiated patients; $p=0.25$ ) (Fig. 1).

\section{Discussion}

The presence of extensive splenomegaly in MF patients undergoing ASCT delays post-transplant granulocyte recovery when compared with those who had splenectomy [12]. The question on the role of splenectomy before ASCT remains open. One should bear in mind an increased mortality and morbidity of splenectomy versus faster post-transplant engraftment. However, in the era of JAK2 inhibitors, the question should soon be unfounded.

The role of splenic irradiation in patients with MF is not well established. It was demonstrated that SI alleviated splenic discomfort and reduced spleen size in a majority of MF patients. Moreover, SI was associated with stabilization or increase of body weight. The median duration of response was 6 months, with maximum duration of response exceeded 41 months. The life-threatening myelosuppression was present in $26 \%$ of the patients and half of them expired [13]. The risk of severe and durable complications of SI remains the main limitation of this procedure, and nowadays it is not routinely advised in daily clinical practice. Total doses of SI are variable and range from 150 to 6500 cGy per course administered in a fractionated manner [2]. Interestingly, 


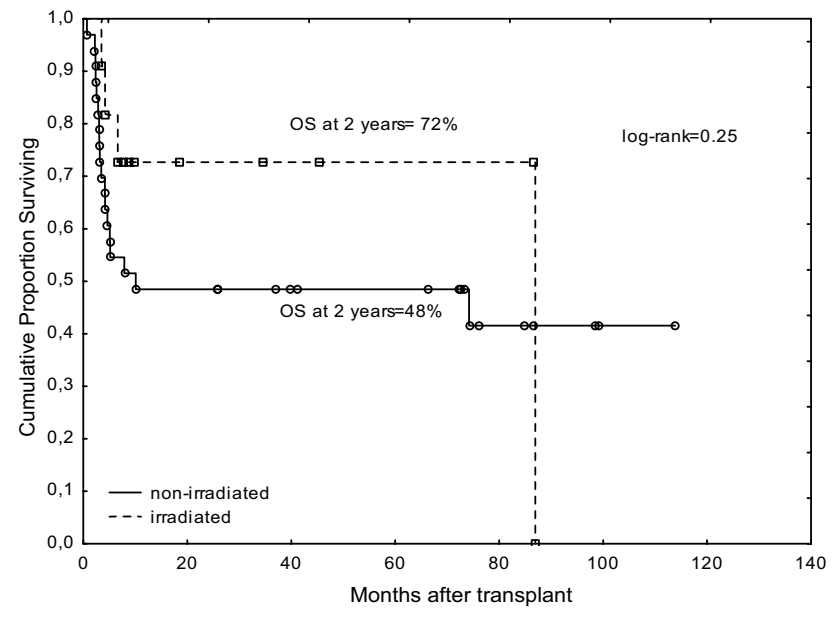

Fig. 1 Comparison of overall survivals between irradiated and nonirradiated patients with myelofibrosis

lower radiation doses (100 cGy in 4 daily doses) administered as an induction-maintenance treatment resulted not only in splenomegaly reduction but also in circulating blast clearance in two patients [14].

Data on the effect of spleen status on outcome after ASCT for myeloproliferative and myelodysplastic disorders have been collected by the Center for International Blood and Marrow Transplant Research (CIBMTR) group. The authors analyzed allografts from a large number of transplanted patients including those with prior splenectomy and splenic irradiation. It was demonstrated that splenectomized patients had faster neutrophil and platelet engraftments when compared with non-splenectomized patients without having an impact on survival. The incidence of acute and chronic GVHD did not differ between irradiated, splenectomized and non-splenectomized patients. Overall mortality rates were also comparable across the groups. However, one should keep in mind that study population was heterogeneous and dose and timing of SI differ between patients. All this may cause difficulties in data interpretation [15].

Taking into account the rarity of data on SI prior to ASCT in MF, we compared the post-transplant outcome between irradiated and non-irradiated patients. We did not observe any difference in engraftment rates, acute and chronic GVHD incidences and survivals between those two analyzed groups. Our study population was homogeneous in terms of diagnosis, conditioning and GVHD prophylaxis. However, there were limitations associated with variations in radiation doses and timing before ASCT. Splenic irradiation is known to result in prolonged pancytopenia with infectious complications, however, that was not a case in our group. A proportion of patients has already been pancytopenic at the time of SI and the median time between SI and ASCT was relatively short (1.5 month). Nevertheless, the incidence of post-transplant infectious complications was comparable between analyzed groups.

The role of SI in leukemic transformation (LT) remains unclear and speculative. It is difficult to conclude whether LT results from prior radiation or represents natural disease course. No difference in LT was present between irradiated and non-irradiated patients in our study.

Reports on the role of SI prior to ASCT for myelofibrosis are sparse and included small groups of patients or case studies $[8,9,16,17]$. Eight patients with MF received median splenic radiation dose of $450 \mathrm{cGy}$ within median of 2 weeks prior to ASCT in Kalman study [8]. All patients had massive pre-transplant splenomegaly $(>20 \mathrm{~cm})$ and achieved a significant decrease in spleen size during repeated assessments. The toxicity of SI was mild. Post-transplant recovery, disease-free and overall survivals were comparable with those who were not irradiated. These results were then confirmed by others. Administration of SI as a part of reduced-intensity conditioning at doses ranging from 300 to $500 \mathrm{cGy}$ in three patients was found to be feasible with acceptable toxicity [9].

\section{Conclusions}

Despite favorable safety profile, splenic irradiation prior to ASCT for myelofibrosis has not beneficial effect on post-transplant outcome and should not be routinely recommended.

\section{Compliance with ethical standards}

Conflict of interest The authors have no competing interest.

Open Access This article is distributed under the terms of the Creative Commons Attribution 4.0 International License (http://creativeco mmons.org/licenses/by/4.0/), which permits unrestricted use, distribution, and reproduction in any medium, provided you give appropriate credit to the original author(s) and the source, provide a link to the Creative Commons license, and indicate if changes were made.

\section{References}

1. Tefferi A. Primary myelofibrosis: 2017 update on diagnosis, risk-stratification, and management. Am J Hematol. 2016;91(12):1262-71.

2. Cervantes F. How I treat splenomegaly in myelofibrosis. Blood Cancer J. 2011;1:e37. https://doi.org/10.1038/bcj.2011.36.

3. Kroger N, Holler E, Kobbe G, et al. Allogeneic stem cell transplantation after reduced-intensity conditioning in patients with myelofibrosis. A prospective, multicenter study of the Chronic Leukemia Working Party of the European Group for Blood and Marrow Transplantation. Blood. 2009;114(26):5264-70.

4. Ballen KK, Shrestha S, Sobocinski KA, et al. Outcome of transplantation for myelofibrosis. Biol Bone Marrow Transplant. 2010;16(3):358-67. 
5. Robin M, Tabrizi R, Mohty M, et al. Allogeneic haematopoietic stem cell transplantation for myelofibrosis: a report of the Societe Francaise de Greffe de Moelle et de Therapie Cellulaire (SFGMTC). Br J Haematol. 2011;152(3):331-9.

6. Robin M, Zine M, Chevret $\mathrm{S}$, et al. The impact of splenectomy in myelofibrosis patients prior to allogeneic hematopoietic stem cell transplantation. Biol Blood Marrow Transplant. 2017;23(6):958-64.

7. Gratwohl A, Iacobelli S, Bootsman N, et al. Splenic irradiation before hematopietic stem cell transplantation for chronic myeloid leukemia: long-term follow-up of a prospective randomized study. Ann Hematol. 2016;95(6):967-72.

8. Kalman NS, Mukhopadhyay ND, Roberts CH, et al. Low-dose splenic irradiation prior to hematopoietic cell transplantation in hypersplenic patients with myelofibrosis. Leuk Lymphoma. 2017;58(12):2983-4.

9. Vyas OH, Kaul E, Rosenberg AS, et al. Splenic irradiation and a reduced-intensity conditioning regimen prior to allogeneic stem cell transplantation for myelofibrosis. Blood. 2014;124:3170.

10. Thiele J, Kvasnicka HM, Tefferi A, et al. Primary myelofibrosis. In: Swierdlow SH, Campo N, Harris NL et al, editors. World health organization classification of tumours of hematopoietic and lymphoid tissues. Lyon: IARC Press; 2008. pp. 44-7.

11. Passamonti F, Cervantes F, Vannucchi AM, et al. A dynamic prognostic model to predict survival in primary myelofibrosis: a study by the IWG-MRT (international working group for myeloproliferative neoplasms research and treatment). Blood. 2010;115(9):1703-8.

12. Li Z, Gooley T, Appelbaum FR, Deeg HJ. Splenectomy and hematopoietic stem cell transplantation for myelofibrosis. Blood. 2001;97:2180-1.

13. Elliot MA, Chen MG, Silverstein MN, Tefferi A. Splenic irradiation for symptomatic splenomegaly associated with myelofibrosis with myeloid metaplasia. Br J Haematol. 1998;103:505-11.

14. Pardanani A, Brown P, Neben-Wittich M, Tobin R, Tefferi A. Effective management of accelerated phase myelofibrosis with low-dose splenic radiotherapy. Am J Hematol. 2010;85(9):715-6.

15. Akpek G, Pasquini MC, Logan B, et al. Effects of spleen status on early outcomes after hematopoietic cell transplantation. Bone Marrow Transplant. 2013;48:825-31.

16. Matsubara E, Yamanouchi J, Kitazawa R, et al. Usefulness of low-dose splenic irradiation prior to reduced-intensity conditioning regimen for hematopoietic stem cell transplantation in elderly patients with myelofibrosis. Case Rep Hematol. 2016. https://doi. org/10.1155/2016/8751329.

17. Ito T, Akagi $\mathrm{K}$, Kondo $\mathrm{T}$, Kawabata H, Ichinohe $\mathrm{T}$, Takaori-Kondo A. splenic irradiation as a component of a reduced-intensity conditioning regimen for hematopoietic stem cell transplantation in myelofibrosis with massive splenomegaly. Tohoku J Exp Med. 2012;228:295-9. 